\title{
Reduced Calibration Curve for Proton Computed Tomography
}

\author{
Olga Yevseyeva ${ }^{\mathrm{a}}$, Joaquim de Assis ${ }^{\mathrm{a}}$, Ivan Evseev ${ }^{\mathrm{b}}$, Hugo Schelin ${ }^{\mathrm{b}}$, Sergei \\ Paschuk $^{\mathrm{b}}$, Edney Milhoretto ${ }^{\mathrm{b}}$, João Setti ${ }^{\mathrm{b}}$, Katherin Díaz ${ }^{\mathrm{c}}$, Joel Hormaza ${ }^{\mathrm{d}}$, \\ and Ricardo Lopes ${ }^{\mathrm{e}}$
}

\begin{abstract}
${ }^{a}$ Instituto Politécnico da UERJ, Rua Alberto Rangel s/no, 28630-050, Nova Friburgo - RJ, BRAZIL ${ }^{b}$ Universidade Tecnológica Federal do Paraná - UTFPR, Av. Sete de Setembro 3166, 80230-901, Curitiba - PR, BRAZIL

${ }^{c}$ Centro de Aplicaciones Tecnologicas y Desarrollo Nuclear-CEADEN, Calle 30 \#502 e/5ta y $7 \mathrm{ma}$ Avenida, Playa, Ciudad Habana, CUBA

${ }^{d}$ Instituto de Biociências da UNESP, Distrito de Rubião Jr. s/no, 18618-000, Botucatu - SP, BRAZIL ${ }^{e}$ Laboratório de Instrumentação Nuclear, COPPE/UFRJ, Av. Horácio Macedo 2030, 21941-914, Rio de Janeiro - RJ BRAZIL
\end{abstract}

\begin{abstract}
The pCT deals with relatively thick targets like the human head or trunk. Thus, the fidelity of pCT as a tool for proton therapy planning depends on the accuracy of physical formulas used for proton interaction with thick absorbers. Although the actual overall accuracy of the proton stopping power in the Bethe-Bloch domain is about $1 \%$, the analytical calculations and the Monte Carlo simulations with codes like TRIM/SRIM, MCNPX and GEANT4 do not agreed with each other. A tentative to validate the codes against experimental data for thick absorbers bring some difficulties: only a few data is available and the existing data sets have been acquired at different initial proton energies, and for different absorber materials. In this work we compare the results of our Monte Carlo simulations with existing experimental data in terms of reduced calibration curve, i.e. the range - energy dependence normalized on the range scale by the full projected CSDA range for given initial proton energy in a given material, taken from the NIST PSTAR database, and on the final proton energy scale - by the given initial energy of protons. This approach is almost energy and material independent. The results of our analysis are important for pCT development because the contradictions observed at arbitrary low initial proton energies could be easily scaled now to typical pCT energies.
\end{abstract}

Keywords: Proton beams, Energy measurement, Calibration curve, Monte Carlo methods. PACS: $24.10 . \mathrm{Lx}$; 02.50.Ng; 02.70.Uu; 05.60.Cd; 07.05.Tp; 81.70.Tx; 82.20.Fd; 87.55.km.

\section{INTRODUCTION}

The interaction of heavy charged particles with thick absorbers has well established analytical description [1]. In addition, the Monte Carlo simulation for this process has a long and successful history [2]. Nevertheless, we have meet the situations while our simulations with such popular codes like TRIM/SRIM [2], MCNPX [3] and GEANT4 [4] do not agreed with each other [5]. It should be stressed that naturally we do not expect an absolute coincidence of the proton spectra generated by such different codes. However, the differences in mean final energy of about $10 \mathrm{MeV}$ for protons with initial energy $250 \mathrm{MeV}$ after $34 \mathrm{~cm}$ water absorber pied our attention [6]. 
A tentative to validate the codes against experimental data for thick absorbers bring us some difficulties: only a few data is available and the existing data sets have been acquired at different initial proton energies, and for different absorber materials. In particular, we succeed to compare TRIM, MCNPX and GEANT4 simulations with the experimental spectra for a number of $\mathrm{Al}$ absorbers with various thicknesses for 19.68 $\mathrm{MeV}$ [7] and 49.1 MeV [8] protons passing through, for two Au targets and 49.1 MeV protons [9], and for polyethylene absorber and $25 \mathrm{MeV}$ protons [10].

In this work we will try to generalize these results to cover the initial proton energy range from approximately $25 \mathrm{MeV}$ up to $250 \mathrm{MeV}$ having in mind the applications for proton computed tomography (pCT) [11]. We hope, however, that our analysis will light up some unobvious nuances in the $\Delta \mathrm{E}-\mathrm{E}$ method exploration as well.

It should be stressed that our analysis is strictly limited to the so-called "BatheBloch region" [1,2], i.e. the formation of the so-called "Bragg peak" is out of our interest. Two criteria were employed to cut the Bragg peak from the analysis: first, the final proton energy after absorber should be above $2 \mathrm{MeV}$, and second, the so-called "Detour factor" predicted by the NIST PSTAR [12] should not be less than 1.00 within $1 \%$ tolerance.

Although the GEANT4 has been validated against the NIST PSTAR stopping powers (SP) for protons [13], and the TRIM use SRIM reference SP table [2], the simulated spectra have significant contradictions with experiment for thick absorbers. It means that in this case the about $1 \%$ accuracy of SPs (for the discussed proton energies) in $[2,12]$ is not enough.

\section{REDUCED CALIBRATION CURVE}

\section{WET Calibration Problem in pCT}

The calibration curve in $\mathrm{pCT}$ is the average residual energy as a function of the proton pass in water for fixed initial energy, which permits to determinate the Water Equivalent Thickness (WET) of any absorber by the measurement of mean outgoing proton energy $[14,15]$. This curve could be easily build basing on NIST PSTAR and SRIM reference tables for the projected ranges, or calculated within the Continuous Slowing Down Approximation (CSDA) [1,2]. However, the problem is that the absolute values will be different depending on the way of curve constructing.

For example, one can easily checkup that the $250 \mathrm{MeV}$ protons will be totally stopped by only $375 \mathrm{~mm}$ of water according to the SRIM, while the NIST PSTAR projected range value is $379 \mathrm{~mm}$. Correspondently, while estimate the final energy of these protons after $30 \mathrm{~cm}$ of water, the difference will be about $2.5 \mathrm{MeV}$, or $2.5 \%$ as the energy is very closed to $100 \mathrm{MeV}$ (See FIGURE 1). It is interesting to note also that the Monte Carlo simulations with GEANT4 and TRIM/SRIM are agreed with NIST PSTAR and SRIM based calibration curves correspondently (See FIGURE 1). It should be noted that for all figures in this work the statistical error bars are smaller than the point dimensions.

This $2.5 \%$ disagreement of these two calibration curves takes place while the differences in total, and (which is practically the same) electronic SP given by reference tables is less than $1.2 \%$ in the proton energy range $100 \div 250 \mathrm{MeV}$. This 
reflects the fact that the measuring of "integrated" energy loss in a thick absorber provides more sensitive test than the measurements of "differential" energy loss in a very thin target.

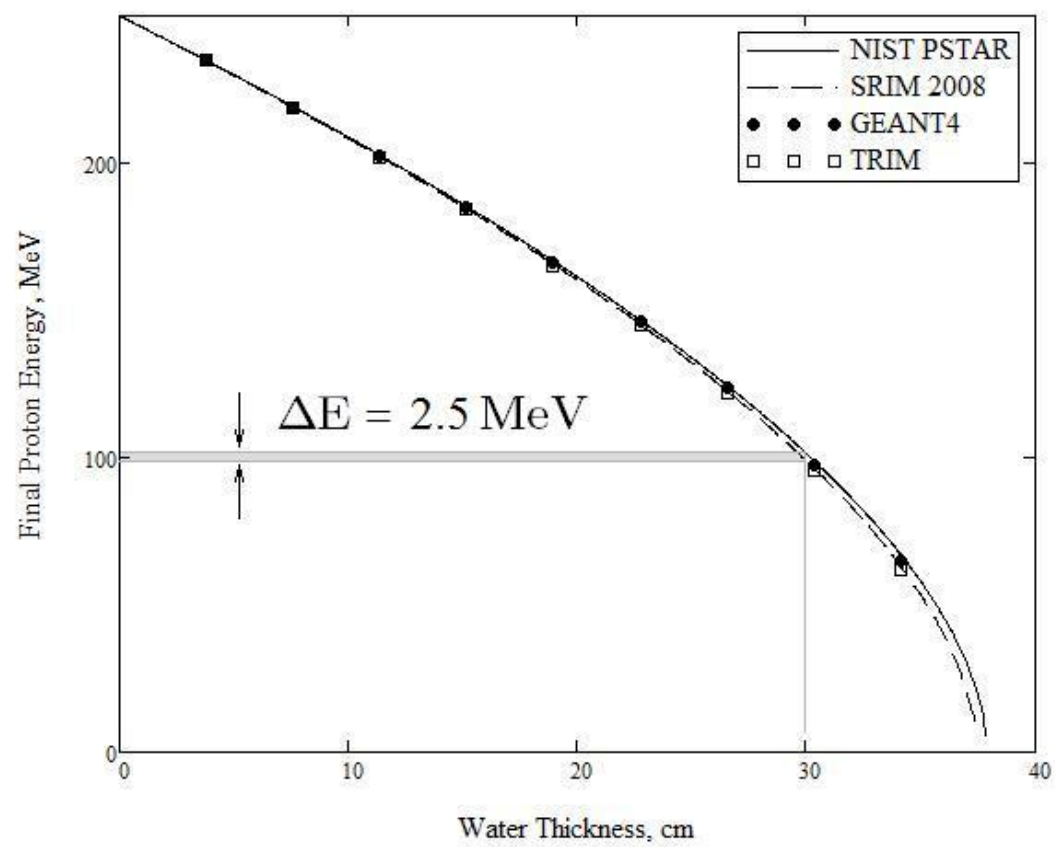

FIGURE 1. The WET calibration curve for $250 \mathrm{MeV}$ protons.

\section{Reduced Calibration Curve}

In experimental practice a solid target is obviously preferable. In literature, there are several experimental data sets obtained on solid targets [16,17]. However, these data sets were obtained for different materials at different initial proton energies. The method we propose to include these data in analysis is to use the reduced calibration curve.

It is easy to show that if one will measure absorber thickness in the full CSDA range units, i.e. as $R / R_{\text {tot }}\left(E_{0}\right)$ ratio, and the outgoing proton energy in the initial energy units, i.e. as $\mathrm{E} / \mathrm{E}_{0}$ ratio, the resulted range - energy dependence (calibration curve) will be almost energy and material independent (See FIGURE 2). It is not absolutely true for any energy and material, but at least for the initial proton energies from approximately $25 \mathrm{MeV}$ to $250 \mathrm{MeV}$ and chemical elements and mixtures with $\mathrm{Z} / \mathrm{A}$ $1 / 2$ it happens within a few percent accuracy.

For instance, there was no any possibility to show separately the calibration curves for $\mathrm{H}_{2} \mathrm{O}, \mathrm{Al}, \mathrm{A}-150$ and $\mathrm{B}-100$ plastics for $25 \mathrm{MeV}$ and $250 \mathrm{MeV}$ protons on FIGURE 2 just because the relative differences are less than $0.5 \%$. There are no visible differences between the NIST PSTAR and SRIM based curves as well, if only the thickness scale is normalized for $\mathrm{R}_{\text {tot }}$ from the corresponding table. 
It is interesting to note that GEANT4 and TRIM/SRIM simulated results for water absorber and $250 \mathrm{MeV}$ protons became agreed with each other and the corresponding calibration curve (Compare FIGURE 1 and FIGURE 2). The experimental data for $\mathrm{Al}$ absorber obtained with $19.68 \mathrm{MeV}$ protons in [16] and for polyethylene at $25 \mathrm{MeV}$ [17] are in a very good agreement with reduced calibration curve on FIGURE 2 as well.

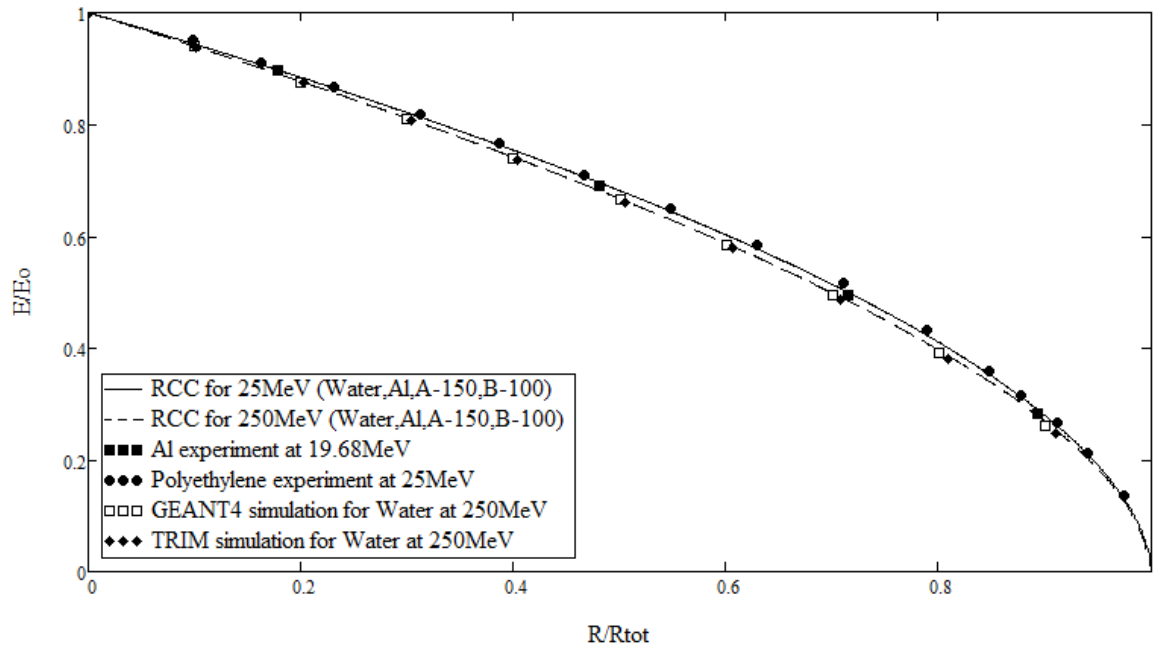

FIGURE 2. The reduced calibration curve for $25 \div 250 \mathrm{MeV}$ protons.

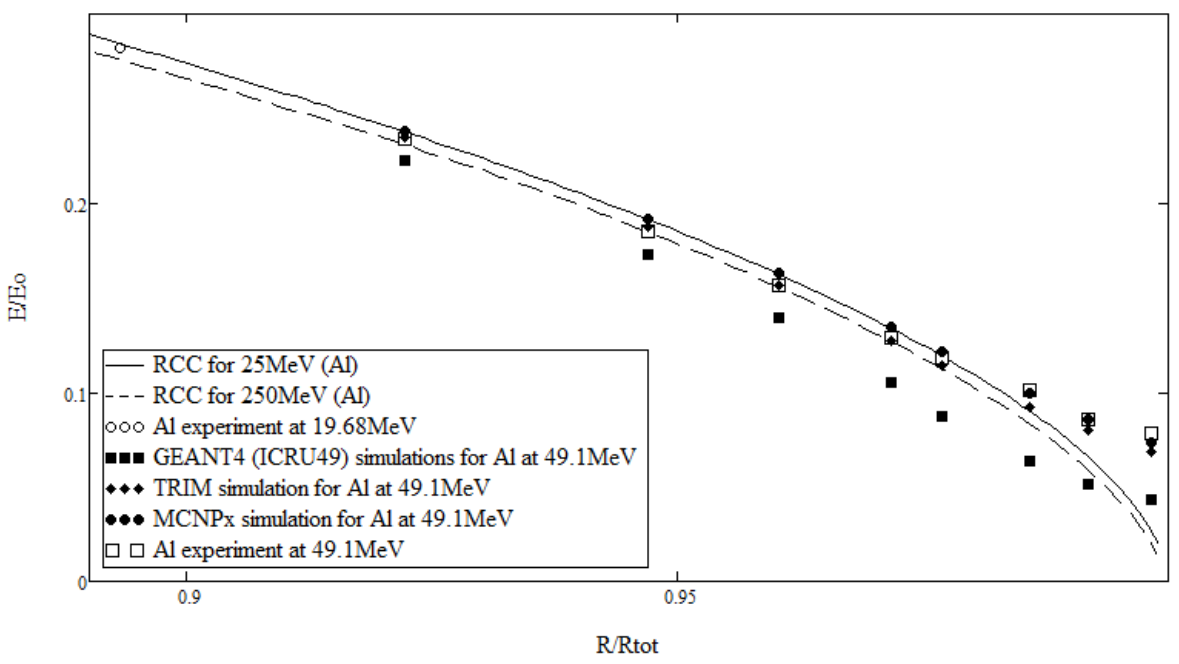

FIGURE 3. The reduced calibration curve for $25 \div 250 \mathrm{MeV}$ protons - only the part of thickest absorbers. 


\section{MONTE CARLO SIMULATIONS AGAINST EXPERIMENT}

Let us now compare our Monte Carlo simulations with existing experimental data for thick Al absorber [16] in the terms of reduced calibration curve (See FIGURE 3). This method of comparison has obvious advantage - the data obtained at $19.68 \mathrm{MeV}$ and $49.1 \mathrm{MeV}$ can now be treated as a unique sequence (the open circus and open boxes at the figure). As the FIGURE 3 just represents the bottom-right part of the FIGURE 2 in an amplified form, but with additional data has not been easy to visualize within the scale of FIGURE 2, it becomes obvious that the $49.1 \mathrm{MeV}$ data for $\mathrm{Al}$ are roughly perfectly falling on the general trend.

It can be seen that our TRIM/SRIM2008 and MCNPX simulations reproduce the experimental data for $49.1 \mathrm{MeV}$ protons. At the same time, the GEANT4 simulation in the standard execution mode does not imitate well the experimental values. Moreover, it is far from the general trend in this case.

\section{CONCLUSIONS}

The reduced calibration curve was proposed as a tool for simultaneous analysis of the experimental data or/and the computer simulations obtained for any initial proton energy from the range $25 \div 250 \mathrm{MeV}$ and any absorber material with $\mathrm{Z} / \mathrm{A} \sim 1 / 2$. The reduced calibration curves were erected from NIST PSTAR and SRIM reference data tables for $\mathrm{H}_{2} \mathrm{O}, \mathrm{Al}, \mathrm{A}-150$ and $\mathrm{B}-100$ absorbers.

The curves were compared with TRIM/SRIM and GEANT4 simulations for water and $250 \mathrm{MeV}$ protons. It was shown that in the reduced form, the noticeable disagreements between NIST PSTAR and SRIM data, and, consequently, between GEANT4 and TRIM/SRIM simulations are generally despairing.

The method was tested involving the experimental data for polyethylene obtained with $25 \mathrm{MeV}$ protons, and $\mathrm{Al}$ absorbers measured with $19.68 \mathrm{MeV}$ and $49.1 \mathrm{MeV}$ protons. The last ones were compared with TRIM/SRIM, MCNPX and GEANT4 simulations.

It was shown that our GEANT4 simulations have a tendency to full down from the general trend with the increasing of the absorber thickness at least for this case. It gives us an additional strong argument to discard absolute error in the energy scale of experiment [16] as a possible reason of disagreement with GEANT4 simulations.

However, the comparison with only a few sets of experimental data was not enough to make a definitive choice for the most adequate way to use GEANT4 for pCT simulations. Thus, we are planning to continue this work involving other experimental data and computer simulations into analysis within the reduced calibration approach.

\section{ACKNOWLEDGMENTS}

The authors are greatly thankful to Dr. Reinhard Schulte from LLUMC (USA) for useful discussions. The work was supported by "Fundação Araucária" (Paraná State, Brazil), CAPES and CNPq. 


\section{REFERENCES}

1. . С. Ремизович, Д. Б. Рогозкин, М. И. Рязанов, ЭЧАЯ, 17(5), 929-981, (1986), in Russian.

2. J. F. Ziegler, J. P. Biersack, M. D. Ziegler, SRIM: The Stopping and Range of Ions in Matter, Chester-MD, SRIM Co., 2008.

3. MCNPX User's Manual (Version 2.4.0), Los Alamos National Laboratory, LA-CP-02-408, USA, 2002.

4. S. Agostinelli, J. Allison, K. Amako, et al., NIM, A506, 250-303, (2003).

5. O. Yevseyeva, "Modelagem Computacional da Tomografia com Feixe de Prótons", Ph.D. Thesis, Universidade do Estado do Rio de Janeiro, 2009, in Portuguese.

6. I. Evseev, J. T. de Assis, O. Yevseyeva, et al., "Comparison of Some Popular Monte Carlo Solutions for Proton Transportation within pCT Problem". In DVD: Proceedings of 2007 International Nuclear Atlantic Conference - INAC 2007, Santos-SP, Brazil. Belo Horizonte-MG, DISCMIDIA, 2007, 1-6.

7. O. Yevseyeva, J. T. de Assis, I. Evseev, et al., "Comparison of GEANT4 Simulations with Experimental Data for Thick Al Absorbers", AIP Conf. Proc. 1139, 97-101 (2009).

8. I. G. Evseev, H. R. Schelin, S. A. Paschuk, et al., "Comparison of SRIM, MCNPX and GEANT simulations with experimental data for thick Al absorbers", Appl. Radiat. Isotopes, (2009), in press.

9. O. Yevseyeva, J. T. de Assis, I. Evseev et al., "Comparison of Some Monte Carlo Simulations for with Proton Experiment for a Thick Au Absorber". In DVD: Proceedings of International Nuclear Atlantic Conference - INAC 2009, Rio de Janeiro-SP, Brazil. ABEN, 2009, 1-4.

10. H. R. Schelin, I. Evseev, S. Paschuk, et al., "The Comparison of GEANT4 with MCNPX and SRIM2008 Monte Carlo Simulations against the Experimental Calibration Curve for Proton Computerized Tomography", to be published.

11. H. F. W. Sadrozinski, V. Bashkirov, B. Keeney, et al., IEEE Trans. Nucl. Sci., 51, 3-9, (2004).

12. http://physics.nist.gov/PhysRefData/Star/Text/PSTAR.html.

13. K. Amako, S. Guatelli, V. N. Ivanchenko, et al., IEEE Trans. Nucl. Sci., 52, 910-918, (2005).

14. R. Schulte, V. Bashkirov, M. C. L. Klock, et al, Med. Phys. 32, 1035-1046, (2005)

15. J. T. de Assis, O. Yevseyeva, I. Evseev, et al., X Ray Spectrometry, 34, 481-492, (2005).

16. C. Tschalär and H. D. Maccabee, Phys. Rev., B1, 2863-2869, (1970).

17. A. Ito and H. Koyama-Ito, Nucl. Instr. and Meth, B3, 584-588, (1984). 
Copyright of AIP Conference Proceedings is the property of American Institute of Physics and its content may not be copied or emailed to multiple sites or posted to a listserv without the copyright holder's express written permission. However, users may print, download, or email articles for individual use. 